\title{
Membership and binarity of solar-type dwarfs in the nearby open cluster Alpha Persei (Mel 20) ${ }^{\star, \star \star}$
}

\author{
J.-C. Mermilliod ${ }^{1}$, D. Queloz ${ }^{2}$, and M. Mayor ${ }^{2}$ \\ ${ }^{1}$ Laboratoire d'Astrophysique, École Polytechnique Fédérale de Lausanne (EPFL), Observatoire, 1290 Sauverny, Switzerland \\ e-mail: jean-claude.mermilliod@obs .unige.ch \\ 2 Observatoire de Genève, 1290 Sauverny, Switzerland
}

Received 21 April 2008 / Accepted 6 June 2008

ABSTRACT

\begin{abstract}
Context. The Alpha Persei open cluster (Melotte 20) is one of the nearby clusters $(d<500 \mathrm{pc})$ for which a long-term systematic radial-velocity monitoring of the solar-type stars has not been published so far.

Aims. We undertook an observing program in 1982 to study the membership, duplicity, and rotation of the main-sequence stars and to compare the results with those of the other nearby clusters.

Methods. Radial-velocity observations of 60 solar-type stars in Mel 20 were obtained with CORAVEL, and of 44 fainter stars with ELODIE spectrographs, in the magnitude range $9.5<V<13.85$

Results. The membership of 37 stars from the list compiled by Heckmann is confirmed according to their proper motions, radial velocities, and photometry. We discovered twelve spectroscopic binaries ( 3 double-lined, 8 single-lined, 1 suspected) among the members and determined an orbit for the double-lined binary He 848. The observed frequency of spectroscopic binaries is therefore $32 \%$ (12/37). Four stars are photometric binaries. In addition, the membership of 33 stars with AP numbers is supported by the present radial velocities. Two single-lined and three double-lined binaries were detected in this sample. The cluster mean radial velocity is $-1.39 \pm 0.17 \mathrm{~km} \mathrm{~s}^{-1}$ based on 18 stars within $2 \sigma$. We found a systematic difference of $3.67 \pm 1.48 \mathrm{~km} \mathrm{~s}^{-1}$ between observedand astrometric radial velocities.

Conclusions. The Alpha Persei cluster members define a tight main sequence in the colour-magnitude diagrams. The remaining scatter observed in the colour-magnitude diagrams and in radial velocities may be due to the difficulty of separating the core Alpha Persei stars from the extended comoving stream.
\end{abstract}

Key words. stars: binary: spectroscopic - Galaxy: open cluster and associations: individual: Alpha Persei (Melotte 20) - techniques: radial velocities

\section{Introduction}

For several years, knowledge of the Alpha Persei cluster (Mel 20), $\alpha=3^{\mathrm{h}} 20^{\mathrm{m}} \cdot 6, \delta=+48^{\circ} 10^{\prime}(\mathrm{J} 2000)$, was based on the $U B V$ study of Mitchell (1960) and the spectral classifications of Morgan et al. (1972) and Abt (1978). These authors used the member selection of Heckmann et al. (1956) and the mainsequence faintest limit was $V=11.7$. Because it is a nearby cluster, $d=190 \mathrm{pc}$, (Robichon et al. 1999), younger than the Pleiades and it contains the F-type supergiant $\alpha$ Persei, Mel 20 is an interesting cluster. Makarov (2006) provides a review of recent literature and a list of 139 members selected on astrometric criteria.

The proper-motion study by Stauffer et al. (1989) permitted him to identify about 30 new low-mass members. UBVRI photometry and radial velocities confirmed the membership of most selected stars. The extensive studies of Prosser (1992, 1994), based on proper motions, photometry, and radial velocities (in most cases only one per star) resulted in the detection of a well-populated, lower main sequence. More recently, Deacon \& Hambly (2004) measured new proper motions on Schmidt

\footnotetext{
* based on observations collected at the Haute-Provence Observatory (France).

$\star \star$ Table 5 is only available in electronic form at the CDS via anonymous ftp to cdsarc.u-strasbg.fr (130.79.128.5) or via http://cdsweb.u-strasbg.fr/cgi-bin/qcat?J/A+A/488/409
}

plates with the SuperCOSMOS facility and published membership probabilities for 302 stars in the magnitude interval $11<R<17.5$, as well as for some 90 AP stars numbered by Prosser (1992, 1994). The Alpha Persei cluster is now a very interesting nearby open cluster with a well-defined main sequence known over a range of magnitudes similar to that of the Pleiades. In addition its distance was determined to a good accuracy from Hipparcos observations (Robichon et al. 1999; van Leeuwen 1999).

Several studies were devoted to the photometric observations of solar-type stars to derive rotational periods from the photometric light curves, by Prosser et al. (1993a,b, 1995), Prosser \& Grankin (1997), O’Dell \& Cameron (1993), O’Dell et al. (1997), Bouvier (1996), and Messina (2001). In total, 94 period determinations for 66 different stars were obtained, providing basic data to study the evolution of rotational velocities and comparing the distribution with those of the Pleiades and younger clusters.

The lower main sequence was investigated by Barrado y Navascués et al. (2002) who searched for brown dwarfs from $J H K$ photometry and determined the mass function. Lodieu et al. (2005) used $R_{\mathrm{c}} I_{\mathrm{c}} K$ photometry to further look for brown dwarfs. They provided a list of 20 additional candidates.

The X-ray observations with the ROSAT satellite, analysed by Randich et al. (1996) and Prosser et al. (1996, 1998a,b) confirmed the membership of many low-mass stars and permitted them to discover new candidates. 
The first comprehensive study of the duplicity of the bright B- and A-type stars was published by Petrie \& Heard (1969), but the number of radial-velocity measurements did not permit them to compute any orbit. The work was taken up again by Morrel \& Abt (1992), who observed 28 of the brightest stars and found only 4 spectroscopic binaries, with periods between 21 and 30 days.

We undertook in 1982 a survey of the solar-type stars in the Alpha Persei cluster as part of our systematic survey of the nearby clusters. But due to the proximity of the Pleiades in the sky and the fact that we gave a higher priority to the latter, the observations of Alpha Persei stars did not progress as fast as desired and we consequently got a smaller number of observations per star.

The main goal was to obtain radial velocities to identify the cluster members, study the binarity, and determine the binary frequency, and also to measure rotational velocities. The observations were more difficult that in the Pleiades due to the larger rotation of the F5/F6 stars in Alpha Persei. Therefore, our study is limited to the sample of stars which could be observed with CORAVEL.

\section{Observations}

\subsection{The sample}

The initial observing programme included 59 F5 - K0 candidates selected on the basis of the $U B V$ photometry published by Mitchell (1960). His selection was based on proper-motion membership from Heckmann et al. (1956). The original limiting magnitude was $B=12.5$. In 1989,18 stars were added to this sample, but only a small number of observations were finally obtained for these stars. Twenty-three F5-F6 stars did not produce any correlation dip because their rotational velocities are larger than about $45 \mathrm{~km} \mathrm{~s}^{-1}$, as is expected for mid-F stars in a young cluster of the age of Alpha Persei. The star He 1000 was observed due to an error on the identification map.

In 1996-1997, 53 additional stars from Prosser's (1992) membership list, as revised by Stauffer et al. (1993), were selected and observed with the ELODIE spectrograph (Baranne et al. 1996) to determine rotational velocities. Stars with only upper limit on $V \sin i$ in the literature, i.e. $V \sin i<10 \mathrm{~km} \mathrm{~s}^{-1}$, were primarily selected for this program. The $V \sin i$ data are analysed in a separate paper (Bouvier et al. 2008).

\subsection{Coravel observations}

The observations were obtained with the CORAVEL radialvelocity scanner (Baranne et al. 1979) installed on the 1-m Swiss telescope at the Haute-Provence Observatory (OHP) for stars later than spectral type F5 and brighter than $B=12.5$. Between November 1982 and December 1994, four to six observations per star were obtained. Binaries were observed more often to derive orbital elements. From July 1989 on, 18 additional stars were added to the sample and observed. One to four observations were obtained.

The radial velocities are on the system defined by Udry et al. (1999) from high-precision radial-velocities obtained with the ELODIE spectrograph. This calibration corrects for most systematic effects of the CORAVEL system. The integration times ranged from about 200 to as much as $1500 \mathrm{~s}$, the average being about $400 \mathrm{~s}$. The errors on well-exposed individual measurements are usually smaller than $0.5 \mathrm{~km} \mathrm{~s}^{-1}$. However, for a few stars with larger rotation $\left(V \sin i \sim 30 \mathrm{~km} \mathrm{~s}^{-1}\right)$ the errors may reach $2 \mathrm{~km} \mathrm{~s}^{-1}$, depending on the width of the correlation function. No corrections for gravitational redshift or convective blueshift have been applied to the observations.

General information and mean radial velocities for the members (Table 1) and non-members (Table 2) are presented separately. Both tables give the identification of Heckmann et al. (1956). A few stars were observed with ELODIE only: He 389, 1101, and 1234 in Table 1 and He 600, 1032, 1086 and 1110 in Table 2.

CORAVEL individual radial velocities will be published in the final catalogue containing 7200 observations for 1253 solartype dwarfs in 13 nearby clusters (Mermilliod et al. 2008a).

\subsection{Elodie observations}

The observations were obtained with the fiber-fed echelle spectrograph ELODIE (Baranne et al. 1996) at the Haute-Provence Observatory $193 \mathrm{~cm}$ telescope during the 1996-1997 season. The echelle spectra are recorded on a $1024 \times 1024$ CCD and the cross-correlation functions are computed by an automatic reduction procedure carried out directly after the observations using a $\mathrm{K} 0$ template. The precision of these observations is estimated to be $0.15 \mathrm{~km} \mathrm{~s}^{-1}$ due to photon noise.

Table 3 collects the mean radial velocities of the stars numbered by Prosser (AP). It contains the identification, the photometric data $\left(V,(B-V),(V-I)_{K}\right)$, the mean radial velocities, error on the mean $\left[\mathrm{km} \mathrm{s}^{-1}\right]$, the number of observations, the radial velocities found in the literature and remarks on duplicity and membership. The individual observations obtained with ELODIE (No, HJD, RV) are given Table 5 available in electronic form only.

\section{Results}

\subsection{Membership}

The determination of the membership of the stars in the field of the Alpha Persei moving cluster is more difficult than in the cases of the Pleiades or Praesepe, although the Alpha Persei cluster is also a nearby aggregate. The location within the MS band is a necessary but not sufficient criterion of membership, because the bright field stars overlap with the cluster's main sequence (MS) within the domain of magnitude under study, as shown by Fig. 4 of Prosser (1992). Some of the members selected on the basis of their proper motions or radial velocities are clearly located outside the MS band and should be rejected from the membership. Membership to the cluster should be accepted only if the three criteria are satisfied.

Radial velocities are necessary but not sufficient to determine the membership of the stars in the cluster field, contrary to what we experienced in other clusters. Although the distribution of the radial velocities of the program stars has a well-defined maximum (Fig. 1), there is a continuum of velocities between -10 and $+10 \mathrm{~km} \mathrm{~s}^{-1}$, and the membership determination is not obvious based on radial velocity only. In addition, small differences around the mean cluster velocity cannot be automatically interpreted as non-membership because of the possible presence of unresolved binaries.

The diameter of the area studied across the sky reaches $6^{\circ}$ and the change of the projection of the space velocity on the line of sight produces a velocity gradient over the cluster. This effect is several times larger than the expected internal velocity dispersion. The gradient is readily computed. The space velocity is given by $V_{\mathrm{s}}=V_{\mathrm{r}}(r)+k \mu d$ where $V_{\mathrm{r}}$ is the radial 
Table 1. Mean radial velocities and general information for 37 members in Melotte 20.

\begin{tabular}{|c|c|c|c|c|c|c|c|c|c|c|c|c|c|c|c|c|c|c|}
\hline $\mathrm{He}$ & $V$ & $B-V$ & $V-I_{k}$ & $U-B$ & Sp.T. & $\mu_{\alpha}$ & $\mu_{\delta}$ & Prob & $V_{\mathrm{r}}$ & $\epsilon$ & $n$ & $\Delta T$ & $P\left(\chi^{2}\right)$ & $V r_{\text {litt }}$ & $N$ & Astr & Diff & Notes \\
\hline 12 & 10.09 & 0.51 & & 0.03 & F6 V & 25.1 & -24.6 & .99 & -0.32 & 2.44 & 5 & 4402 & 0.001 & -1.2 & 1 & 2.11 & 1.78 & SB? \\
\hline 135 & 9.71 & 0.49 & & 0.01 & F5 V & 23.5 & -23.8 & .99 & -2.43 & 0.37 & 4 & 2567 & 0.615 & -1.0 & 1 & 1.88 & 4.31 & \\
\hline 174 & 11.62 & 0.95 & 0.85 & & & 27.2 & -25.1 & .99 & -1.54 & 0.22 & 4 & 2629 & 0.383 & -2.4 & 2 & 2.25 & 3.79 & $\mathrm{dbl}$ \\
\hline 270 & 10.11 & 0.51 & & 0.01 & F7 V & 22.6 & -22.0 & .79 & +0.74 & 1.77 & 6 & 4402 & 0.000 & +0.1 & 1 & 2.42 & 1.68 & SB \\
\hline 299 & 11.19 & 0.64 & 0.56 & & F7 & 18.5 & -19.0 & .87 & -1.82 & 0.48 & 5 & 4104 & 0.019 & -2.1 & 1 & 2.10 & 3.92 & \\
\hline 309 & 9.96 & 0.49 & & 0.01 & F5 V & 23.4 & -21.4 & .86 & +3.84 & 3.37 & 1 & & & +3.1 & 1 & 3.60 & -0.24 & \\
\hline 334 & 10.33 & 0.56 & 0.45 & 0.04 & F7 V & 22.5 & -23.1 & .00 & -2.47 & 0.72 & 6 & 4405 & 0.004 & -2.2 & 2 & 3.94 & 6.41 & \\
\hline 338 & 9.93 & 0.56 & & 0.02 & F7 V & 26.5 & -26.8 & .96 & +0.78 & 3.14 & 1 & & & +1.2 & 1 & 3.75 & 2.97 & $\mathrm{dbl}$ \\
\hline 350 & 11.13 & 0.71 & & & G3 & 23.2 & -23.0 & .99 & -0.55 & 1.16 & 5 & 5081 & 0.175 & & & 4.19 & 4.74 & \\
\hline 361 & 9.68 & 0.44 & & 0.01 & F4 V & 22.9 & -22.5 & .69 & -2.43 & 0.77 & 4 & 2889 & 0.745 & +0.5 & 1 & 3.22 & 5.65 & \\
\hline 389 & 11.17 & 0.67 & 0.55 & & G0 & 22.5 & -23.9 & .99 & -1.96 & 0.10 & 2 & 14 & 0.479 & & & 2.50 & 4.46 & \\
\hline 453 & 10.39 & 0.62 & 0.53 & 0.12 & F8 V & 22.8 & -23.5 & .00 & -5.25 & 6.81 & 3 & 711 & 0.000 & -1.7 & 2 & 3.44 & 8.69 & SB \\
\hline 490 & 9.56 & 0.45 & 0.31 & 0.04 & F3 IV-V & 23.1 & -25.6 & .99 & -1.65 & 0.52 & 4 & 2885 & 0.182 & -1.4 & 2 & 2.89 & 4.54 & \\
\hline 601 & 11.40 & 0.74 & 0.59 & 0.20 & & 22.9 & -24.1 & .00 & -0.84 & 0.26 & 5 & 4065 & 0.960 & -0.9 & 1 & & & \\
\hline 621 & 9.86 & 0.49 & & 0.02 & F4 V & 22.5 & -27.0 & .96 & -0.51 & 0.67 & 5 & 4113 & 0.480 & -1.7 & 2 & 3.33 & 3.84 & \\
\hline 660 & 10.06 & 0.56 & 0.50 & 0.11 & F5 V & 23.1 & -25.5 & .99 & -0.88 & 1.18 & 6 & 4406 & 0.064 & -1.0 & 2 & 2.64 & 3.52 & $\mathrm{dbl}$ \\
\hline 684 & 10.54 & 0.56 & 0.45 & 0.04 & F9 V & 20.6 & -25.8 & .98 & +2.77 & 0.60 & 2 & 4810 & 0.000 & -1.4 & 1 & 3.25 & 0.48 & SB \\
\hline 696 & 11.42 & 0.74 & 0.65 & 0.18 & & 19.8 & -25.0 & .89 & -0.62 & 0.26 & 6 & 4065 & 0.221 & -1.1 & 1 & & & \\
\hline 733 & 9.94 & 0.50 & & 0.04 & F6 V & 22.1 & -26.6 & .99 & +1.79 & 1.59 & 6 & 4338 & 0.021 & +0.6 & 1 & 3.31 & 1.52 & \\
\hline 750 & 10.54 & 0.59 & & 0.09 & F9 V & 22.0 & -25.6 & .99 & -0.64 & 0.57 & 5 & 4109 & 0.295 & +0.6 & 1 & 3.26 & 3.90 & \\
\hline 767 & 10.66 & 0.60 & & 0.08 & F9 V & 21.1 & -26.0 & .95 & -0.97 & 0.33 & 5 & 4109 & 0.066 & -1.5 & 3 & 2.98 & 3.95 & \\
\hline 794 & 10.06 & 0.55 & 0.46 & 0.07 & F3 IV-V & 21.4 & -24.8 & .93 & +2.51 & 3.85 & 4 & 358 & 0.000 & +2.5 & 1 & 2.82 & 0.31 & SB \\
\hline 828 & 11.59 & 0.71 & 0.64 & & F8 & 19.4 & -26.2 & .98 & +0.60 & 0.27 & 4 & 2890 & 0.420 & +1.0 & 1 & & & \\
\hline 833 & 10.03 & 0.49 & 0.42 & 0.03 & F6 V & 22.5 & -27.0 & .99 & +0.33 & 0.83 & 8 & 4109 & 0.025 & +0.4 & 2 & & & \\
\hline 848 & 9.99 & 0.59 & 0.46 & 0.07 & F9 V & 22.2 & -26.4 & .99 & -0.26 & 0.22 & 41 & 4115 & 0.000 & +3.1 & 2 & 3.66 & 3.92 & SB2O \\
\hline 859 & 10.48 & 0.56 & 0.48 & 0.02 & G0 & 23.6 & -7.1 & .00 & +0.72 & 0.91 & 10 & 5225 & 0.000 & +4.8 & 1 & & & SB \\
\hline 863 & 9.21 & 0.52 & & 0.02 & F6 V & 20.8 & -27.2 & .99 & -23.30 & 4.81 & 1 & & & -7.0 & 1 & 3.37 & & SB \\
\hline 917 & 11.10 & 0.70 & 0.57 & 0.19 & F4 & 23.9 & -28.3 & .99 & -0.08 & 1.12 & 5 & 4022 & 0.095 & +1.0 & 1 & & & \\
\hline 968 & 10.41 & 0.57 & & 0.03 & F8 V & 21.1 & -27.6 & .99 & +0.88 & 0.88 & 6 & 4107 & 0.221 & -3.3 & 3 & 3.67 & 2.79 & \\
\hline 988 & 9.80 & 0.56 & & 0.07 & F5 IV-V & 26.8 & -38.0 & .99 & +2.45 & 0.32 & 5 & 4109 & 0.249 & +1.8 & 1 & & & $\mathrm{dbl}$ \\
\hline 992 & 10.84 & 0.72 & 0.64 & 0.23 & G0 & 15.3 & -11.0 & .00 & & & 1 & & & -5.2 & 1 & & & SB2 \\
\hline 1086 & 11.32 & 0.67 & 0.58 & & G0 & 20.7 & -24.7 & .99 & -1.52 & 0.15 & 1 & & & -1.0 & 1 & 2.90 & 4.42 & \\
\hline 1101 & 11.25 & 0.69 & 0.62 & & G4 & 20.9 & -28.5 & .99 & -0.33 & 0.87 & 3 & 1924 & 0.368 & -2.4 & 1 & 3.36 & 3.69 & \\
\hline 1144 & 11.32 & 0.73 & & & G5 & 32.3 & -12.4 & & -6.57 & 0.15 & 1 & & & +9.7 & 1 & & & SB \\
\hline 1180 & 10.07 & 0.49 & 0.37 & & F7 & 20.9 & -27.9 & .99 & -0.34 & 2.35 & 4 & 2983 & 0.004 & & & 3.81 & 4.25 & \\
\hline 1185 & 11.19 & 0.72 & & 0.19 & F7 & 19.9 & -27.9 & .99 & -2.15 & 0.97 & 8 & 5138 & 0.000 & & & 3.84 & 5.99 & SB \\
\hline 1234 & 10.81 & 0.72 & 0.61 & & G2 & 21.4 & -33.7 & .98 & & & 1 & & & +5.7 & 1 & 2.82 & & SB2 \\
\hline
\end{tabular}

Notes: He: identification of Heckmann et al. (1956); the $V,(B-V),(V-I)_{K}$ and $(U-B)$ photometry from WEBDA or Prosser et al. (1996, 1998a,b); Sp.T.: MK spectral type; $\mu_{\alpha}, \mu_{\delta}$ : UCAC2 proper motions, Prob: membership probabilities of Fresneau (1980); $V_{\mathrm{r}}$ : the mean radial velocities; $\epsilon$ : error on the mean; $n$ : number of observations; $\Delta T$ : time interval of the observations; $P\left(\chi^{2}\right)$ : probability $P\left(\chi^{2}\right) ; V r_{\text {litt }}$ : radial velocities from Stauffer et al. $(1985,1989)$ or Prosser $(1992,1994)$; $N$ : number of spectra; Astr: astrometric radial velocity (Makarov 2006); Diff: difference with the observed radial velocity. Notes: remarks on duplicity and membership (SB: spectroscopic binary, SB2: double-lined binary; SB2O: double-lined binary with an orbit; dbl: photometric binary).

velocity in $\mathrm{km} \mathrm{s}^{-1}, k$ is the usual conversion factor $(k=4.738)$, $\mu$ is the absolute proper motion in arcseconds per year, and $d$ is the distance in parsecs. The values adopted here are: $V_{\mathrm{r}}=$ $-1.39 \mathrm{~km} \mathrm{~s}^{-1}, \mu=0.03416 \mathrm{yr}^{-1}$ and $d=185 \mathrm{pc}$. These numbers give a space velocity of $+29.83 \mathrm{~km} \mathrm{~s}^{-1}$ and an angle at the cluster centre between the space and radial velocities of $\lambda=92^{\circ} 40$. The radial velocity gradient is given by $d V_{\mathrm{r}}=-V_{\mathrm{s}} d \lambda \sin \lambda$ where $d \lambda$ is the angular diameter of the cluster (taken here as $6^{\circ}$ ). This leads to a radial-velocity difference of $3.12 \mathrm{~km} \mathrm{~s}^{-1}$ across the cluster area.

Therefore, we also had to rely on UCAC2 proper motions (Zacharias et al. 2004) to finalise the selection. Hipparcos mean proper motion for Alpha Persei is $\mu_{\alpha} \cos \delta=+22.93$ mas yr$^{-1}$ and $\mu_{\delta}=-25.56$ mas yr$^{-1}$ (Robichon et al. 1999). These values are close to the mean values derived from UCAC2 data for 17 non-binary members: $\mu_{\alpha} \cos \delta=+22.3 \pm 1.1(\mathrm{rms})$ mas $\mathrm{yr}^{-1}$ and $\mu_{\delta}=-25.4 \pm 2.1 \mathrm{mas} \mathrm{yr}^{-1}$ and to those computed from Tycho- 2 from the same 17 stars: $\mu_{\alpha} \cos \delta=+21.6 \pm 2.7$ (rms)

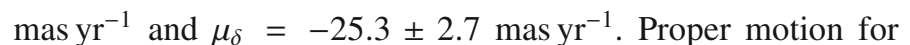
He 462 is taken from TYCHO-2 catalogue (Hog et al. 2000).

The general agreement of the TYCHO-2 and UCAC2 proper motions is good. However, in the region of Alpha Persei, the UCAC2 proper motions are better than those of TYCHO-2 for $\mu_{\alpha}$ because the UCAC2 dispersion given above is 2.5 times smaller than that of TYCHO-2. The improvement is evident in a vector-point diagram. The $\mu_{\delta}$ are also slighty better, although the UCAC2 proper motions show a larger dispersion on $\mu_{\delta}$ than on $\mu_{\alpha}$. Half of the spectroscopic and photometric binaries show discrepant proper motions, viz., He 12, 174, 338 859, 988, 992, 1144,1234 , while their radial velocities support membership and they are normally located within the MS band. These stars are represented by crosses in Fig. 2. Determination of their systemic velocities and reexamination of their proper motions would be necessary to better constrain their membership.

Stars with radial velocities comprised in the interval -5.0 to $+5.0 \mathrm{~km} \mathrm{~s}^{-1}$ were selected in the first step, then their proper motions were plotted in a $\left(\mu_{\alpha} \cos \delta, \mu_{\delta}\right)$ diagram (Fig. 2). Finally, 
Table 2. Non-members from radial velocity and proper motions, or from the colour-magnitude diagrams

\begin{tabular}{|c|c|c|c|c|c|c|c|c|c|c|c|c|c|c|c|c|}
\hline $\mathrm{He}$ & $V$ & $B-V$ & $V-I_{k}$ & $U-B$ & Sp.T. & $\mu_{\alpha}$ & $\mu_{\delta}$ & Prob & $V_{\mathrm{r}}$ & $\epsilon$ & $n$ & $\Delta T$ & $P\left(\chi^{2}\right)$ & $V r_{\text {litt }}$ & $N$ & Notes \\
\hline 28 & 10.64 & 0.75 & & 0.30 & G0 V & 36.9 & -21.8 & .00 & +4.71 & 0.18 & 5 & 4459 & 0.800 & +5.1 & 2 & \\
\hline 29 & 10.62 & 0.60 & & 0.10 & F8 IV-V & 16.5 & -36.9 & .99 & +23.33 & 0.47 & 5 & 1534 & 0.013 & +22.2 & 1 & \\
\hline 143 & 10.47 & 0.71 & & 0.22 & F8 IV-V & 11.2 & -22.1 & & +5.26 & 0.14 & 18 & 747 & 0.000 & -3.6 & 1 & SB1O \\
\hline 201 & 9.85 & 0.60 & & 0.11 & F8 V & 42.6 & -21.8 & .00 & +6.21 & 0.22 & 6 & 4459 & 0.158 & +6.1 & 1 & \\
\hline 271 & 10.41 & 0.60 & & 0.09 & F7 & 13.9 & -11.5 & .00 & +5.32 & 0.90 & 6 & 5523 & 0.250 & +7.1 & 1 & \\
\hline 276 & 9.93 & 0.64 & & 0.16 & F8 V & 8.8 & -17.1 & .48 & -9.83 & 0.21 & 7 & 4460 & 0.784 & -7.8 & 1 & \\
\hline 290 & 10.71 & 0.64 & & 0.15 & F9 V & 27.9 & -18.6 & .20 & +6.69 & 0.79 & 7 & 4460 & 0.002 & +6.6 & 1 & \\
\hline 340 & 11.45 & 0.69 & & 0.17 & F9 & 13.5 & -23.6 & & -7.69 & 0.15 & 6 & 4107 & 0.228 & -8.6 & 2 & \\
\hline 347 & 10.51 & 0.68 & & 0.18 & G0 V & 28.0 & -13.4 & .00 & +8.67 & 0.36 & 6 & 4463 & 0.013 & +8.8 & 1 & \\
\hline 416 & 10.85 & 0.68 & & & F6 & 5.4 & -17.4 & & +9.39 & 0.40 & 1 & & & +8.9 & 2 & \\
\hline 435 & 9.86 & 0.61 & & 0.18 & F6 IV & 9.9 & -21.5 & .49 & -18.15 & 2.33 & 1 & & & -15.1 & 2 & \\
\hline 444 & 11.16 & 0.83 & 0.75 & & G3 & 4.6 & -15.1 & .00 & -52.75 & 3.36 & 8 & 1448 & 0.000 & & & SB \\
\hline 457 & 11.70 & 0.74 & 0.68 & 0.27 & & 7.8 & -15.0 & .00 & -3.29 & 0.23 & 25 & 4064 & 0.000 & & & $\mathrm{SB} 1 \mathrm{O}, \mathrm{cmd}$ \\
\hline 462 & 11.40 & 0.83 & & 0.28 & G0 & 20.9 & -4.9 & & +27.69 & 0.69 & 3 & 1060 & 0.000 & +11.6 & 1 & SB \\
\hline 517 & 10.19 & 0.51 & & & F5 V & 2.5 & -28.4 & .00 & +14.49 & 1.02 & 7 & 792 & 0.010 & & & \\
\hline 538 & 11.61 & 0.66 & 0.65 & 0.28 & A7 & 24.3 & -17.5 & & +3.02 & 0.40 & 1 & & & +0.9 & 2 & $\mathrm{cmd}$ \\
\hline 551 & 11.17 & 0.65 & 0.56 & 0.14 & F8 & 18.7 & -16.8 & .23 & +20.49 & 0.28 & 5 & 4108 & 0.412 & +20.0 & 1 & \\
\hline 573 & 9.47 & 0.49 & 0.40 & & F4 & 7.7 & -42.9 & .83 & -15.43 & 0.59 & 1 & & & +1.6 & 1 & SB \\
\hline 600 & 11.90 & 0.68 & 0.61 & 0.27 & & 12.5 & -1.0 & .00 & -5.20 & 0.15 & 1 & & & -2.8 & 1 & $\mathrm{cmd}$ \\
\hline 615 & 11.06 & 0.90 & 0.87 & & G3 & 7.6 & -1.8 & & -37.87 & 8.25 & 3 & 382 & 0.000 & & & SB \\
\hline 633 & 10.25 & 0.56 & 0.51 & & F8 IV-V & 32.8 & -29.6 & .99 & +23.23 & 0.47 & 6 & 4108 & 0.008 & +22.9 & 1 & \\
\hline 771 & 11.10 & 0.68 & 0.66 & 0.12 & G0 & 20.6 & -20.2 & .00 & +18.83 & 0.28 & 4 & 1532 & 0.567 & & & \\
\hline 1000 & 11.38 & & & & $\mathrm{~F} 1$ & 51.8 & -40.4 & & +18.02 & 0.32 & 4 & 1533 & 0.947 & & & \\
\hline 1032 & 11.11 & 0.66 & 0.54 & & F5 & 13.2 & -18.9 & .88 & & & 1 & & & & & SB2 \\
\hline 1036 & 11.34 & 0.83 & 0.64 & 0.52 & & 28.2 & -34.8 & .00 & +32.93 & 0.39 & 1 & & & +32.7 & 1 & \\
\hline 1100 & 11.20 & 0.80 & & 0.35 & G3 & 31.0 & -22.3 & .00 & +14.96 & 0.20 & 5 & 3983 & 0.030 & +15.6 & 2 & \\
\hline 1110 & 11.67 & 0.71 & 0.72 & & G3 & 3.3 & -23.9 & .51 & +0.81 & 0.15 & 1 & & & +1.0 & 2 & $\mathrm{cmd}$ \\
\hline 1181 & 10.57 & 0.58 & 0.49 & & $\mathrm{G} 2$ & 31.8 & -14.8 & .98 & -4.77 & 0.21 & 4 & 2983 & 0.178 & -4.5 & 2 & \\
\hline 1187 & 10.06 & 0.55 & & 0.09 & F6 IV-V & 16.0 & -22.3 & .00 & +15.68 & & 12 & 1539 & 0.000 & & & SB2 \\
\hline
\end{tabular}

Notes: same format as Table 1. "cmd" means that the star is judged non-member from its position in the $(V, B-V)$ colour-magnitude diagrams.

stars located within the MS band, obtained for $E(B-V)=0.09$, a value derived from photoelectric $U B V$ data (Meynet et al. 1993) and $m-M=6.60$ were retained as members.

The selection of cluster members made on the basis of the proper motions and radial velocities is confirmed by the two colour-magnitude diagrams, $V$ vs. $B-V$ (Fig. 3 ) and $V$ vs. $V-I_{K}$ (Fig. 4). Black dots represent the members and open circles, non-members. These two plots show that the selection based on the $U B V$ photoelectric data (stars brighter than $V=11.5$, i.e. CORAVEL sample) contains a large fraction of non-members, while the selection for ELODIE, based on photometry, proper motion and radial velocities is much cleaner. The main sequence of Alpha Persei is well defined. The $\left(V, V-I_{K}\right)$ sequence presents more scatter than the $(V, B-V)$ plane because the $\left(V-I_{K}\right)$ index is more sensitive than $(B-V)$ to the presence of late-type companions, which emit more in the $I$ band.

The final selection corresponds to proper motions within $2 \sigma$ of the mean value, with the notable exception of the above mentionned binaries, which have proper motions outside this limit. Orbital periods are not known and we therefore cannot decide if the orbital motion altered the proper-motion determination or not.

\subsubsection{Members}

The final selection of members and candidate members is listed in Table 1. The proper motions from the UCAC2 catalogue (Zacharias et al. 2004) support the membership of several stars for which Fresneau's (1980) membership probability is zero, as for example stars He 334, 453. A few stars deserve additional comments.

Stars He 299, 828, and 1086 are located about $0.15-0.20$ mag below the main sequence, but their proper motions and radial velocities are in good agreement with the cluster mean values. The depth effect could explain their position. A radius of $10 \mathrm{pc}$ produces a maximum difference on the individual distance modulus of $0.12 \mathrm{mag}$, which is close to the size of the observed scatter around the main sequence.

Star He 863 has only one CORAVEL radial-velocity observation, at $-23 \mathrm{~km} \mathrm{~s}^{-1}$ and three from Petrie \& Heard (1969) at $-13,-12$ and $+5 \mathrm{~km} \mathrm{~s}^{-1}$. It is considered as a probable member because the proper motion supports its membership. Its position in the colour-magnitude diagram implies that it is either a double, or even a triple, system or a non-member. Further observations will be necessary to ascertain its membership.

Four stars, He 135, 309, 334 and 98, have radial velocities slightly differing from the mean value for the cluster. The first one, He 135, is about 0.3 mag above the ZAMS and the difference between CORAVEL and literature values may support its binary character. Additional observations would be useful. Two, He 309 and 334, are located on the "single" star sequence and the CORAVEL and literature velocities are in good agreement. Finally, He 988 is clearly a photometrically-detected binary located about 0.6 mag above the ZAMS. Several stars in the Pleiades (Mermilliod et al. 1992) and Praesepe (Mermilliod \& Mayor 1999) were also considered to be binary members from their position within the MS band, although no radial-velocity variability was observed. Only an upper limit for the X-ray flux of He 988 is available, $\log L_{\mathrm{X}}<28.85$ (Randich et al. 1996). 
Table 3. Elodie radial velocities.

\begin{tabular}{rccccccccc}
\hline \hline AP & $V$ & $B-V$ & $V-I_{\mathrm{k}}$ & $V_{\mathrm{r}}$ & $\epsilon$ & $n$ & $V r_{\text {litt }}$ & $N$ & $\mathrm{Rem}$ \\
\hline 14 & 11.94 & 0.83 & 0.68 & -0.85 & 0.15 & 1 & -0.1 & 1 & \\
25 & 12.25 & 0.88 & 0.75 & -1.51 & 0.15 & 1 & -0.1 & 1 & \\
28 & 13.09 & 1.05 & 0.97 & -0.39 & 0.15 & 1 & -0.1 & 1 & \\
33 & 12.94 & 1.04 & 0.88 & -0.19 & 0.15 & 1 & +0.5 & 1 & \\
41 & 12.03 & 0.85 & 0.71 & -0.59 & 0.15 & 1 & +0.6 & 1 & \\
65 & 13.00 & 1.05 & 0.91 & -0.23 & 0.15 & 1 & -0.4 & 1 & \\
70 & 12.83 & 1.00 & 0.88 & -0.31 & 0.15 & 1 & -0.1 & 1 & \\
72 & 12.78 & 0.99 & 0.87 & -0.31 & 0.15 & 1 & -0.2 & 1 & \\
75 & 13.82 & 1.27 & 1.41 & +4.63 & 0.15 & 1 & -6.0 & 1 & $\mathrm{SB}$ \\
89 & 12.31 & 0.85 & 0.74 & +2.02 & 0.15 & 1 & +2.2 & 1 & $\mathrm{M} ?$ \\
97 & 12.08 & 0.87 & 0.75 & -1.41 & 2.29 & 2 & +5.0 & 1 & $\mathrm{SB}$ \\
98 & 12.80 & 1.00 & 0.86 & -0.89 & 0.15 & 1 & -1.0 & 1 & \\
101 & 13.85 & 1.25 & 1.24 & -1.99 & 0.15 & 1 & -1.7 & 1 & \\
102 & 11.96 & 0.80 & 0.69 & -0.94 & 0.15 & 1 & -2.1 & 1 & \\
104 & 12.06 & 0.78 & 0.66 & +0.89 & 0.15 & 1 & +0.8 & 1 & \\
106 & 12.96 & 1.01 & 0.90 & -0.90 & 0.15 & 1 & -1.4 & 1 & \\
110 & 12.27 & 0.92 & 0.81 & -0.78 & 0.15 & 1 & -0.5 & 1 & \\
114 & 13.30 & 1.08 & 1.06 & -0.61 & 0.15 & 1 & +0.3 & 1 \\
121 & 11.89 & 0.79 & 0.70 & -1.47 & 0.15 & 1 & -2.2 & 1 & $\mathrm{M} ?$ \\
156 & 11.89 & & 0.73 & -4.00 & 0.15 & 1 & -10.4 & 1 & $\mathrm{SB} ?$ \\
158 & 11.93 & 0.85 & 0.82 & -0.87 & 0.15 & 1 & -2.1 & 1 & \\
168 & 11.98 & 0.90 & 0.75 & +6.11 & 0.15 & 1 & +5.0 & 1 & $\mathrm{NM}$ \\
169 & 13.28 & & 1.07 & +0.21 & 0.15 & 1 & -0.4 & 1 & \\
173 & 12.26 & & 0.88 & & & 1 & & 1 & $\mathrm{SB} 2$ \\
194 & 12.02 & 0.74 & 0.66 & -4.82 & 0.15 & 1 & -5.4 & 1 & $\mathrm{NM}$ \\
196 & 12.51 & & 0.82 & +0.12 & 0.15 & 1 & +0.2 & 1 & \\
197 & 12.31 & 1.00 & 0.98 & -1.03 & 0.15 & 1 & -1.0 & 1 & $\mathrm{dbl}$ \\
200 & 12.65 & & 1.01 & +3.26 & 0.15 & 1 & & 1 & $\mathrm{SB} 2$ \\
201 & 13.08 & & 1.04 & +1.07 & 0.15 & 1 & -0.5 & 1 & \\
206 & 12.68 & & 0.82 & +14.97 & 0.15 & 1 & +15.4 & 1 & $\mathrm{NM}$ \\
212 & 13.24 & & 0.97 & -0.40 & 0.15 & 1 & -0.9 & 1 & \\
213 & 11.55 & 0.83 & 0.74 & & & 1 & & 1 & $\mathrm{SB} 2$ \\
235 & 13.12 & 1.03 & 1.02 & +1.57 & 0.15 & 1 & +1.1 & 1 & \\
252 & 12.95 & 1.12 & 1.05 & -3.77 & 0.15 & 1 & -4.2 & 1 & $\mathrm{NM} ?$ \\
255 & 12.18 & & 0.70 & +2.12 & 0.15 & 1 & +2.8 & 1 & $\mathrm{M} ?$ \\
256 & 11.79 & 0.81 & 0.72 & +1.34 & 0.15 & 1 & +0.8 & 1 & \\
257 & 13.00 & & 0.92 & +0.35 & 0.15 & 1 & +0.7 & 1 & \\
\hline & & & & & & & & & \\
& & & & &
\end{tabular}

The star He 1144 is considered to be a binary star because one ELODIE observation at $V_{\mathrm{r}}=-6.6 \mathrm{~km} \mathrm{~s}^{-1}$ differs by $16.3 \mathrm{~km} \mathrm{~s}^{-1}$ from that of Prosser (1992), $+9.7 \mathrm{~km} \mathrm{~s}^{-1}$. It lies on the "single" star sequence. Two stars (AP 173 and 213) were detected as double-lined binaries from one ELODIE observation. The present list of members agrees well with the membership determinations published by Stauffer et al. (1985) and Prosser (1992). Differences concern stars detected as new binaries.

\subsubsection{Non-members}

Parameters for stars judged non-members are listed in Table 2. The format is similar to that of Table 1. Most stars are clearly non-members on the basis of their radial velocities. Star He 1000 was observed due to an error on the identification map.

Four stars (He 457, 538, 600, 1110) have radial velocities that could support their membership, but are located more than 0.3 mag below the main sequence in Fig. 3, (open squares) and their proper motions differ notably from the cluster mean motion. Accordingly, they are declared non-members. This is indicated by the comment "cmd" in Table 2. They seem to be at larger distances, but share the apparent motion of the cluster and could be outer members of the moving cluster. He 573 is also rejected from membership on the basis of its proper motion. It

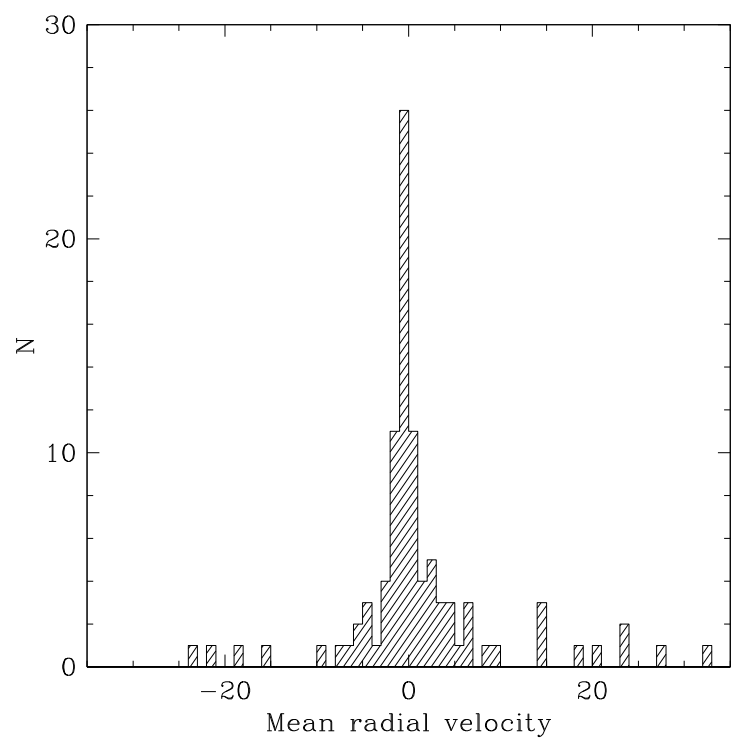

Fig. 1. Distribution of the mean radial velocities.

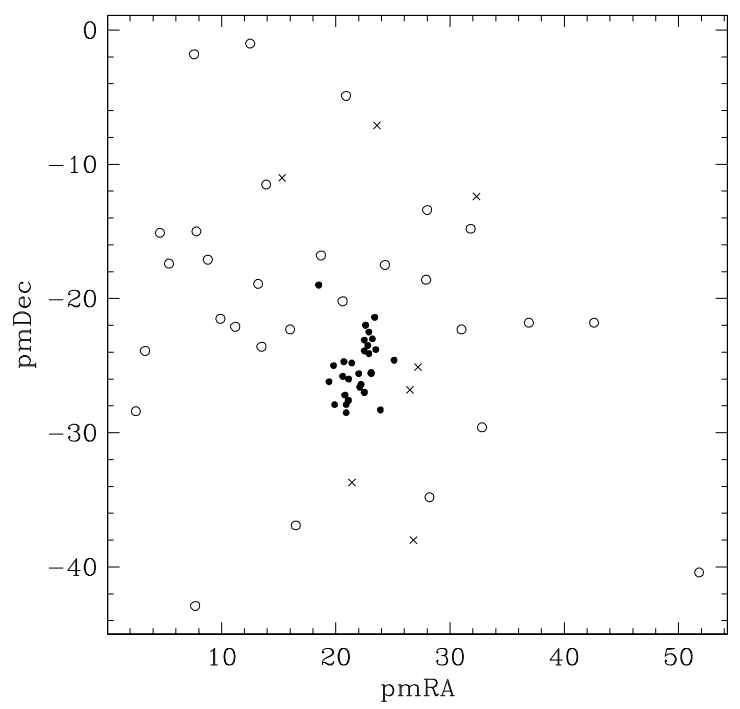

Fig. 2. Distribution of the proper motions. Black dots stand for retained members on the basis of the 3 criteria: proper motions, radial velocities, and photometry. Crosses indicate probable binary members and open circles, non-members.

could be a binary according to the difference between the literature and CORAVEL observations, which corresponds to its location in the $V, B-V$ colour-magnitude diagram.

He 615 deserves a special note. It was suggested by Prosser because it was detected in X-ray. However neither the proper motion nor the photometry clearly supported its membership. The present radial velocities are also against membership. Prosser obtained two observations at -37 . and $-20 \mathrm{~km} \mathrm{~s}^{-1}$ about one year apart (Julian dates not known) and suspected this star was a binary. Our three observations confirm the binarity.

The fact that a few stars have membership probabilities larger than $90 \%$, but have radial velocities or a position in the colour-magnitude diagrams in disagreement with membership stresses again that all three criteria are necessary to constrain the membership of stars to open clusters. 


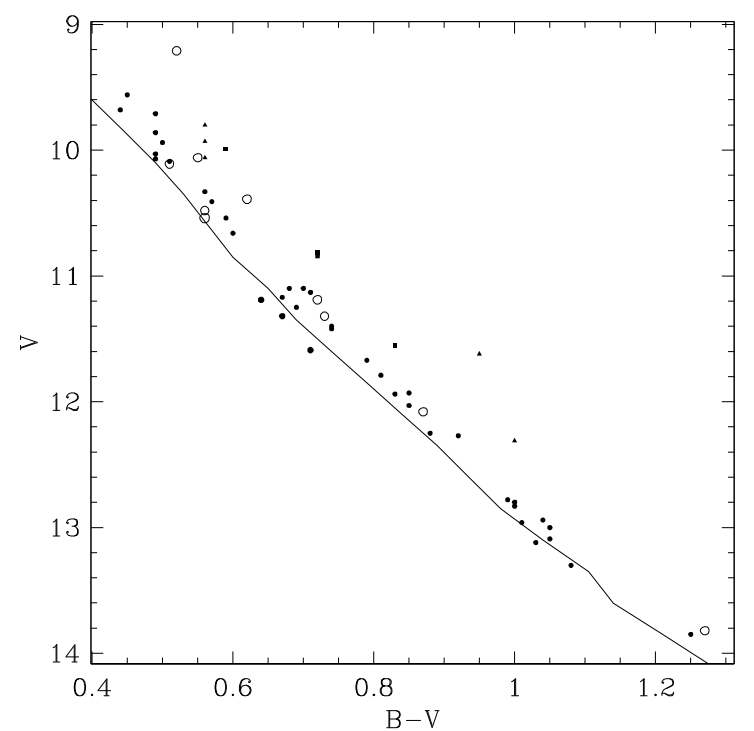

Fig. 3. Colour-magnitude diagram. Symbols as in Fig. 2. The stars with the best kinematical parameters define a narrow sequence. Open squares indicate stars judged as non-members on the basis of their position below the ZAMS of Mermilliod (1981) plotted with $E(B-V)=0.09$ and $m-M=6.60$.

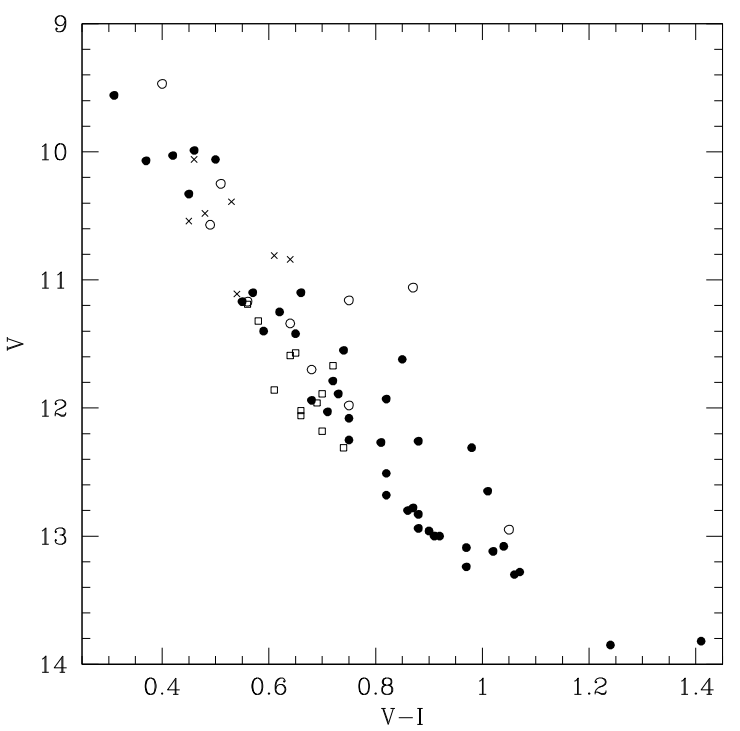

Fig. 4. Colour-magnitude diagram. Symbols as in Fig. 2.

\subsubsection{AP-numbered stars}

Because a selection of best candidate members was applied to form the sample, most stars labelled AP are probably bona fide members. The ELODIE radial velocities are, in most cases, in very good agreement with those published by Stauffer et al. (1985) and Prosser $(1992,1994)$. As discussed in Sect. 3.1, the exact limit beyond which a star can be classified as non-member with certainty is a bit fuzzy.

A difference of several $\mathrm{km} \mathrm{s}^{-1}$ have been found for AP 75, 97,156 and these stars are strongly suspected of being spectroscopic binaries. Stars AP 173, 200 and 213 happen to be doublelined binaries. Due to the small number of observations available, the detection of binaries is most probably incomplete and the AP-numbered stars will not be included in the discussion of binary frequency.

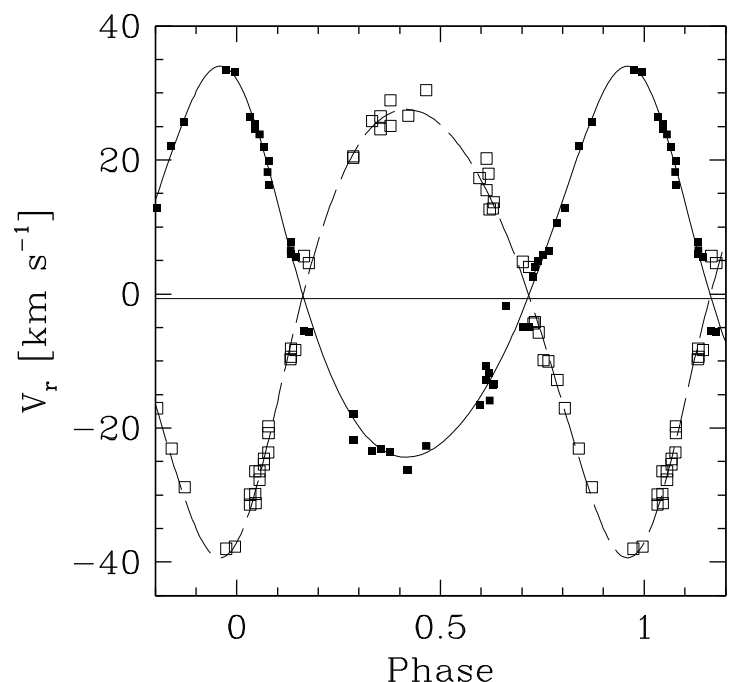

Fig. 5. Radial-velocity curve for the double-lined binary member He 848.

The membership of a few stars can be questioned on the basis of these results: AP 168, 194, 206, and 252 are most probably non-members of the central cluster, according to the discussion made for the brighter stars. AP 89, 121, and 255 are possible non-members.

\subsection{Spectroscopic binaries}

Eleven, possibly twelve, spectroscopic binaries were detected among the sample selected from Heckmann et al. (1956) catalogue (Table 1). This results in a minimum frequency of $32 \%$. This value can be compared to the frequencies obtained in the central part of the Pleiades, $11 / 88=13 \%$, for $P<1000$. (Mermilliod et al. 1992); Praesepe, 16/80 $=20 \%$ (Mermilliod \& Mayor 1999); and in Coma Berenices, 7/32 $=22 \%$ (Mermilliod et al. 2008c). Only one orbit could be determined, for the doublelined binary He 848 (Fig. 5). The orbital elements are given in Table 4 . The binary frequency may be revised to a lower level if some of the binaries with deviating proper motions proved to be non-members.

Among the 29 stars classified as non-members, five, perhaps six, are binaries and two orbits were determined for He 143 and He 457 and are presented in Figs. 6 and 7. The orbital elements are also given in Table 4 . The proper motions indicate that both stars are non-members. The systemic velocity of $\mathrm{He} 143$ differ by $6.53 \mathrm{~km} \mathrm{~s}^{-1}$ from the mean cluster velocity and it lies close to the binary ridge $0.75 \mathrm{mag}$ above the main sequence although it is a SB1. The expected secondary has not been observed. The systemic velocity of $\mathrm{He} 457$ differs from the cluster mean value by only $2.02 \mathrm{~km} \mathrm{~s}^{-1}$, but it is located below the ZAMS. Hence, for both stars, none of the three criteria is fully satisfied.

Star He 1187, a double-lined binary, deserves a specific comment. Although orbital elements cannot be determined with the available observations, even with the help of published radial velocities, the systemic velocity and mass ratio can nevertheless be computed according to the method designed by Wilson (1941). The pairs of velocities $u_{n}, v_{n}$ from several spectra define a straight line with a negative slope in the $u, v$ plane. This slope corresponds to the mass ratio $M_{B} / M_{A}$, since $\frac{\mathrm{d} v}{\mathrm{~d} u}=-r$ according to Wilson's Eq. (2). If $u_{0}$ and $v_{\mathrm{o}}$ are the intercepts of the straight line on the axes, then $r=\frac{v_{0}}{u_{0}}$ and $\gamma=\frac{u_{0} v_{0}}{u_{0}+v_{0}}$. Seven velocity pairs yielded the relation $v=-0.952 u+30.604$. Hence, the mass ratio 
Table 4. Orbital elements of 3 spectroscopic binaries.

\begin{tabular}{lccc}
\hline \hline Element & He 848 & He 143 & He 457 \\
\hline$P[\mathrm{~d}]$ & 89.7734 & 6.1458 & 202.38 \\
& 0.0087 & .0011 & 0.14 \\
[HJD-2 440 000] & 45038.39 & 45994.781 & 45868.7 \\
$e$ & 0.67 & 0.063 & 3.2 \\
& 0.180 & 0.095 & 0.327 \\
$\gamma\left[\mathrm{km} \mathrm{s}^{-1}\right]$ & 0.010 & 0.006 & 0.026 \\
& -0.21 & +5.26 & -3.29 \\
$\omega\left[{ }^{\circ}\right]$ & 0.20 & 0.14 & 0.23 \\
& 19.2 & 133.4 & 328.1 \\
$K_{1}\left[\mathrm{~km} \mathrm{~s}^{-1}\right]$ & 2.9 & 4.2 & 5.5 \\
& 29.52 & 31.46 & 11.21 \\
$K_{2}\left[\mathrm{~km} \mathrm{~s}^{-1}\right]$ & .54 & 0.20 & 0.30 \\
& 33.53 & & \\
$f(m)\left[M_{\odot}\right]$ & 0.50 & & \\
& 0.228 & 0.01960 & 0.0250 \\
$a \sin i[\mathrm{Gm}]$ & 0.014 & 0.00041 & 0.0027 \\
$\sigma(\mathrm{O}-\mathrm{C})\left[\mathrm{km} \mathrm{s}{ }^{-1}\right]$ & 35.85 & 2.646 & 29.5 \\
$n_{\text {obs }}$ & 0.72 & 0.019 & 1.1 \\
& 1.81 & 0.56 & 1.06 \\
& 41 & 18 & 25 \\
\hline
\end{tabular}

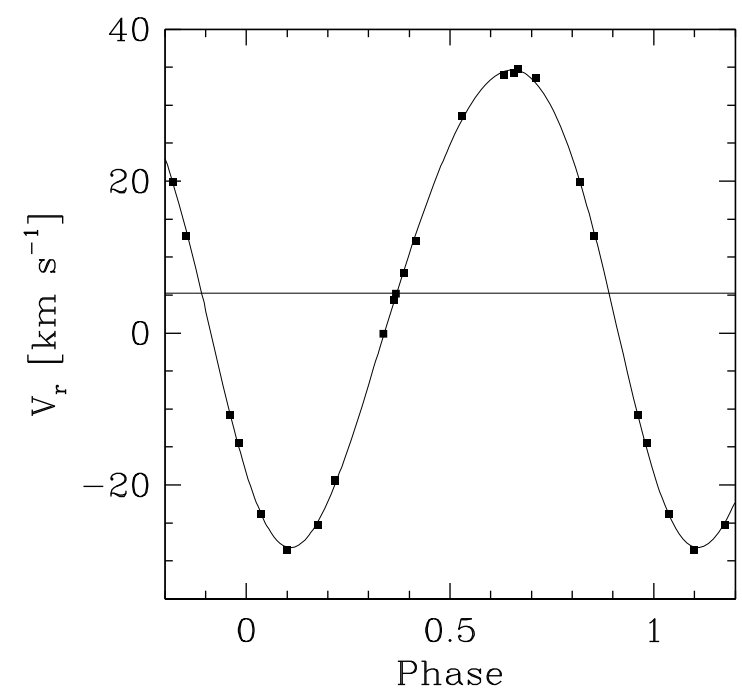

Fig. 6. Radial-velocity curve for the non-member He 143.

is equal to 0.95 and the systemic velocity is $+15.7 \mathrm{~km} \mathrm{~s}^{-1}$. This system is therefore classified as non-member.

\subsection{Cluster mean velocity}

With the 23 stars with constant velocities and the systemic velocity of $\mathrm{He} 848$, we obtain a value of $-1.27 \pm 0.23 \mathrm{~km} \mathrm{~s}^{-1}$ (s.e. of the mean), with a rms $=1.08 \mathrm{~km} \mathrm{~s}^{-1}$ for the mean velocity of the Alpha Persei cluster. If stars He 135, 309, 334, 361, and 988 are omitted at the level of $2 \sigma$, the mean velocity becomes $-1.39 \pm 0.17 \mathrm{~km} \mathrm{~s}^{-1}$ (s.e. of the mean), with an observed $\mathrm{rms}=0.70 \mathrm{~km} \mathrm{~s}^{-1}$ for 18 stars. The mean velocity is not much affected, but the velocity dispersion is evidently reduced. The values of the velocity dispersion is in agreement with the upper limit of $1.1 \mathrm{~km} \mathrm{~s}^{-1}$ determined by Makarov (2006) from proper motions.

Our mean velocity is in agreement with the value published by Stauffer et al.(1993), $\sim-2 \mathrm{~km} \mathrm{~s}^{-1}$. It confirms, if necessary, the membership of the F5Ib supergiant $\alpha$ Per itself, $V_{\mathrm{r}}=-2.04 \pm$ 0.05 , based on 106 measurements (Mermilliod et al. 2008b). The

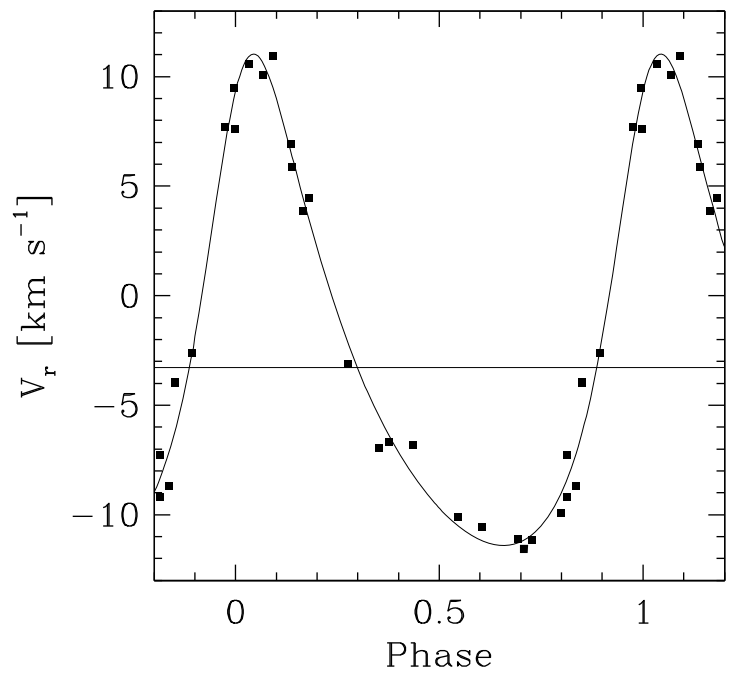

Fig. 7. Radial-velocity curve for the non-member He 457.

difference, $(-1.27)-(-2.04)=0.77 \mathrm{~km} \mathrm{~s}^{-1}$ can be interpreted as due to the difference in gravitational redshift between the dwarfs and the supergiant because the fixed mask in CORAVEL makes no allowance for the change in gravitational redshift for stars of various luminosity classes. In velocity unit, the gravitational redshift is given by

$R V_{\mathrm{g}}=0.64(M / R)$

with $M$ and $R$ in solar unit. Following Nordström et al. (1997), we have $M / R \sim 0.9$ for the $\mathrm{G}$ dwarfs. We computed $R V_{\mathrm{g}} \sim 0.05$ for the supergiant $\alpha$ Per. The difference is then $\sim 0.85$, close to the observed value.

\section{Comparison with astrometric radial velocities}

Makarov (2006) published a kinematical study of the open cluster Alpha Persei and computed astrometric radial velocities. His results for the members of the Alpha Persei cluster are reproduced in the column (Astr) in Table 1. Differences with the observed radial velocities are given in column (Diff). For stars not flagged as binaries, these differences vary from -0.24 to $6.61 \mathrm{~km} \mathrm{~s}^{-1}$, with a mean value of $3.67 \pm 1.48 \mathrm{~km} \mathrm{~s}^{-1}$. The gravitational redshift is less than $1 \mathrm{~km} \mathrm{~s}^{-1}$ as computed in Sect. 3.3 and cannot account for this difference. The astrometric radial velocities are sensitive to the exact position of the convergent point as discussed by Makarov (2006).

\section{Colour-magnitude diagram}

The $V, B-V$ colour-magnitude diagram (Fig. 8) of the cluster members selected from Tables 1 and 3 presents a main sequence well-defined by the single members (filled circles). Several spectroscopic single-lined binaries (open circles) are closely located on the single-star locus (He 12, 859, 684, 1185, 1144, AP 97 and 75), while three are clearly above this locus (He 863, 794 and 453). Double-lined binaries (filled squares) are well above the ZAMS as expected (He 848, 1234, 992, AP 213). Finally, five stars are clearly members according to their kinematical data, but are located above the single-star sequence (He 988, 338, 660, 174, AP 197). They are therefore considered photometric binaries (filled triangles) and are identified by the remark "dbl" in the last column of Table 1. A number of stars from Table 3 are missing in this diagram because the $B-V$ colour indices are not available. 


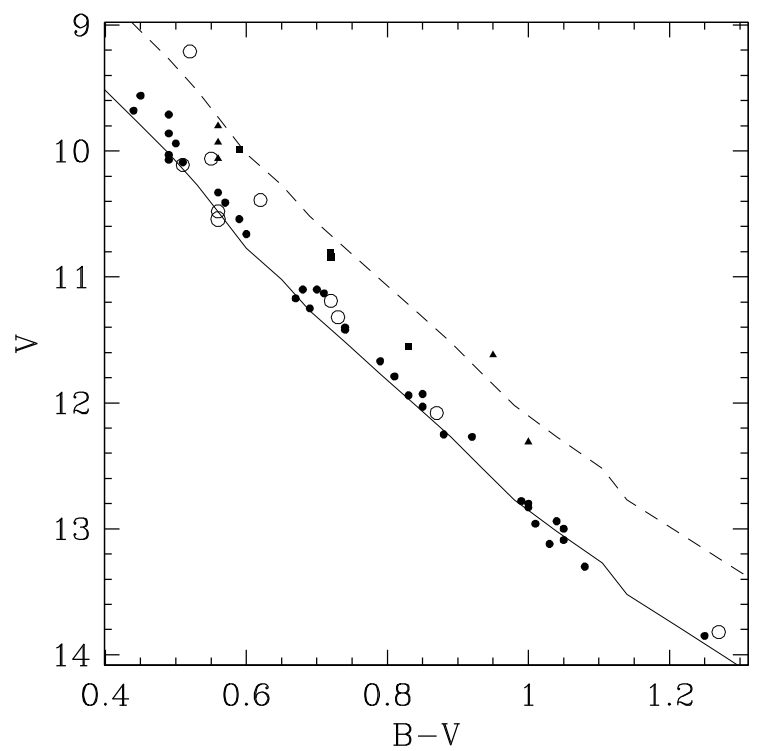

Fig. 8. Colour-magnitude diagram of the selected members. The stars with the best kinematical parameters define a narrow sequence. Symbols are: filled circles, single members; open circles, singlelined binaries (SB1); filled squares, double-lined binaries (SB2); triangles, photometric binaries (dbl). The sequences are the ZAMS (lower, continuous curve) and the binary ridge (upper, dashed curve) (Mermilliod 1981).

\section{Conclusions}

The original list of candidate stars for the study of the Alpha Persei cluster was based on the photometric membership derived from the analysis of the $(V, B-V)$ colour-magnitude diagram. Refined membership criteria based on radial-velocity observations and available proper motions showed that nearly half of the stars located within the MS band are in fact nonmembers. After a very severe selection, the final set of cluster members shows very similar kinematical characteristics and define a tight main sequence in the colour-magnitude diagrams. The membership of 28 stars has been confirmed and further 9 stars are strong candidate members. Depth effects may be visible in the $(V, B-V)$ colour-magnitude diagram, but are less evident in $(V, V-I)$.

Our selection was compared with the membership results of Prosser $(1992,1994)$ based on radial velocities, lithium presence and $\mathrm{H} \alpha$ emission and is found in very good agreement. The larger number of radial velocities obtained with CORAVEL permitted us to solve a number of ambiguities, mostly concerning suspected binaries. The selection of cluster members in the Alpha Persei cluster proved to be more delicate than in the Pleiades and Praesepe clusters for which radial velocities show less dispersion and offer a better clear-cut membership criterion.

It should be stressed that the area covered by the Heckmann et al. (1956) original study is not centered on the cluster centre, but on the brightest star, $\alpha$ Persei itself, which is somewhat at the border of the cluster core. Therefore the south and east parts of the cluster area are not completely surveyed. The present percentage of binaries may be biaised.
Due to the way the catalogue of cluster members has been established in the Alpha Persei cluster, from proper motions, radial velocities, photometry, X-ray fluxes, spectroscopic criteria on lithium and $\mathrm{H} \alpha$ emission over various areas and to different limiting magnitudes, conclusions on the cluster size and luminosity function are beyond the scope of the present paper. Additional observations would be necessary to form a more complete and representative sample and to settle the remaining cases with ambiguous membership.

\section{References}

Abt, H. A. 1978, PASP 90, 692

Bouvier, J., Queloz, D., Mermilliod, J.-C., \& Mayor, M. 2008, A\&A, in preparation

Barado y Navascués, D., Bouvier, J., Stauffer, J. R., Lodieu, N., \& McCaughrean, M. J. 2002, A\&A, 396, 813

Baranne, A., Mayor, M., \& Poncet, J.-L. 1979, Vistas Astron., 23, 279

Baranne, A., Queloz, D., Mayor, M., et al. 1996, A\&AS, 119, 373

Bouvier, J. 1996, A\&AS, 120, 127

Deacon, N. R., \& Hambly, N. C. 2004, A\&A, 416, 125

Fresneau, A. 1980, AJ, 85, 66

Heckmann, O., Dieckvoss, W., \& Kox, H. 1956, AN, 283, 109

Hog, E., Fabricius, C., Makarov, V. V., et al. 2000, A\&A, 355, L27

Lodieu, N., McCaughrean, M. J., Barado y Navascués, D., Bouvier, J., \& Stauffer, J. R. 2005, A\&A, 436, 853

Makarov, V. V. 2006, AJ, 131, 2967

Mermilliod, J.-C. 1981, A\&A, 97, 235

Mermilliod, J.-C., \& Mayor, M. 1999, A\&A, 352, 479

Mermilliod, J.-C., \& Paunzen, E. 2003, A\&A, 410, 511

Mermilliod, J.-C., Rosvick, J. M., Duquennoy, A., \& Mayor, M. 1992, A\&A, 265,513

Mermilliod, J.-C., Mayor, M., \& Udry, S. 2008a, submitted

Mermilliod, J.-C., Mayor, M., \& Udry, S. 2008b, in press

Mermilliod, J.-C., Grenon, M., \& Mayor, M. 2008c, A\&A, submitted

Messina, S. 2001, A\&A, 371, 1024

Meynet, G., Mermilliod, J.-C., \& Maeder, A. 1993, A\&AS, 98, 477

Mitchell, R. I. 1960, ApJ, 132, 68

Morgan, W. W. Hiltner, W. A., \& Garrison, R. 1972, AJ, 76, 242

Morrell, N., \& Abt, H. A. 1992, ApJ, 393, 666

Nordström, B., Andersen, J., \& Andersen, M. I. 1997, A\&A, 322, 460

O’Dell, M. A., \& Cameron, A. C. 1993, MNRAS, 262, 521

O'Dell, M. A., Hilditch, R. W., \& Cameron, A. C. 1997, MNRAS, 284, 874

Petrie, R. M., \& Heard, J. F. 1969, PDAO, 14, 319

Prosser, C. F. 1994, AJ, 107, 1422

Prosser, C. F. 1995, AJ, 103, 488

Prosser, C. F., \& Grankin, K. N. 1997, Publ. LA Noe Obs., 1, 3

Prosser, C. F., \& Randich, S. 1998a, AN, 319, 201

Prosser, C. F., Shetrone, M. D., Marilli, E., et al. 1993a, PASP, 105, 1407

Prosser, C. F., Schild, R. E., Stauffer, J. R., \& Jones, B. F. 1993b, PASP, 105, 269

Prosser, C. F., Shetrone, M. D., Dasgupta, A., et al. 1995, PASP, 107, 211

Prosser, C. F., Randich, S., Stauffer, J. R., Schmitt, J. H. M. M., \& Simon, T. 1996, AJ, 112, 1570

Prosser, C. F., Randich, S., \& Simon, T. 1998b, AN, 319, 215

Randich, S., Schmitt, J. H. M. M., Prosser, C. F., \& Stauffer, J. R. 1996, A\&A, 305,785

Robichon, N., Arenou, F., Mermilliod, J.-C., \& Turon, C. 1999, A\&A, 345, 471

Stauffer, J. R., Hartmann, L. W., Burnham, J. N., \& Jones, B. F. 1985, ApJ, 289, 247

Stauffer, J. R., Hartmann, L. W., \& Jones, B. F. 1989, ApJ, 346, 160

Stauffer, J. R., Prosser, C. F., Giampapa, M. S., Soderblom, D. R., \& Simon, T. 1993, AJ, 106, 229

Udry, S., Mayor, M., \& Queloz, D. 1999, in Precise Stellar Radial Velocities, ed.

J. B. Hearnshaw, \& C. D. Scarfe, IAU Coll., 170, ASP Conf. Ser., 185, 367

van Leeuwen, F. 1999, A\&A, 341, L71

Wilson, O. C. 1941, ApJ, 93, 29

Zacharias, N., Urban, S. E., Zacharias, M. I., et al. 2004, AJ, 127, 3043 